\title{
Ideen zur Veranschaulichung von Aussagen mit Modalverben Sprachwissenschaftliche Annahmen - für den Unterricht aufbereitet
}

\author{
Michael Richter (Lüneburg)
}

\begin{abstract}
The topic of the present paper is the relation between linguistics and language education. Put as a question one could ask: 'what benefit can linguistics have for language teaching?'. It will be claimed that as a precondition, language teachers should have more than just marginal insight into theories of natural language. I will try to support this postulate using sentences with modal verbs: based on a certain linguistic theory, illustrations of sentences with modal verbs will be given. These illustrations are thought to be a first step for a didactic preparation (transformation) of explanations given by a linguistic theory for natural language phenomena.

As a consequence of the considerations it will be postulated that it is obligatory for the education of teachers to include linguistic modules, since, in line with the hypothesis of this paper, insight into linguistic theories can help:

1) to understand phenomena of natural language and

2) to find a didactical preparation for the phenomena in question.
\end{abstract}

\section{$1 \quad$ Einleitung}

In diesem Aufsatz möchte ich eine theoretische Vorstellung von Modalverben anschaulich machen. Ziel ist es, Anregungen für eine sprachdidaktische Umsetzung zu geben.

Anhand der Beispiele soll 1. meine Hypothese bekräftigt werden, dass Vertrautheit der Lehrenden mit linguistischen Theorien hilfreich für eine Vermittlung des entsprechenden sprachlichen Sachverhalts im Unterricht sein kann und 2. gezeigt werden, wie grammatisches Wissen vernetzt werden kann: Modalität wird nicht isoliert, sondern in einem Anwendungskontext betrachtet.

Der Aufbau des Aufsatzes ist wie folgt: Zuerst wird eine Standortbestimmung wissenschaftlicher und pädagogischer Grammatiken gegeben bzw. das gegenseitige Wirkungsverhältnis skizziert. In Abschnitt 3.1 werden einige Einwände gegen (deduktiven) Grammatikunterricht kurz diskutiert, wobei ich insbesondere der Hypothese widersprechen möchte, dass grammatisches Wissen "Träges Wissen" sei. Theoretisches Wissen, didaktisch 
aufbereitet und reduziert, kann, wie ich meine, gut zu der Bildung von grammatischem Wissen und damit zum Verständnis von Sprache beitragen.

Die Abschnitte 4 und 5 enthalten eine sehr knappe theoretische Einordnung der Modalverben und die, ebenfalls sehr knappe, Vorstellung einer sprachwissenschaftlichen Theorie, deren Grundannahmen mir für die o.g. Ziele geeignet erscheinen. Die Veranschaulichung der theoretischen Annahmen findet der Leser in Abschnitt 6.

\section{Wissenschaftliche Grammatik und didaktische Grammatik}

Eine wissenschaftliche Grammatik strebt danach, zu generalisieren, d.h. sie fasst ein beobachtetes Einzelphänomen als Teil eines größeren Systems auf. Der umfassendste Kontext ist die Gesamtheit der natürlichen Sprachen, deren Universalien wissenschaftliche Grammatiken aufspüren und formalisieren wollen. Ziel ist es, von Einzelphänomenen zu abstrahieren und eine große Menge sprachlicher Phänomene mit so wenig Regeln wie möglich zu erfassen. Wissenschaftliche Grammatiken bedienen sich einer formalen Sprache, um die Darstellung übersichtlich, überschau- und nachvollziehbar sowie eindeutig zu machen. Man denke beispielsweise an Phrasenstrukturregeln, an Merkmalstrukturen von lexikalischen Elementen oder auch an Regeln oder Beschränkungen, wie sie in modernen Grammatiktheorien verwendet werden: Zur Beschreibung dieser Dinge ist natürliche Sprache schwerlich geeignet.

Was sind die Anwendungsgebiete der wissenschaftlichen Grammatik? In der heutigen Zeit muss an prominenter Stelle die KI-Forschung genannt werden. Hier geht es um Erkennung und Generierung von Sprache, was u.a. von Interesse für Kommunikation mit Maschinen und Automaten ist. Z.T. werden probabilistische Verfahren in die maschinelle Sprachverarbeitung miteinbezogen, z.B. bei der Erkennung von Wortgrenzen und Wortarten.

Eine didaktische Grammatik dagegen sucht nicht in erster Linie nach Sprachuniversalien. Ihr Anwendungsgebiet ist der gesteuerte Sprachunterricht in einer Fremd- oder in der Muttersprache. Die didaktische Grammatik, wobei man hier z.B. an die traditionelle Schulgrammatik denken kann, strebt nicht in erster Linie danach, sprachliche Phänomene zu erklären, wie es eine wissenschaftliche Grammatik tut, sondern hier geht es oft um klare Regeln, die insbesondere beim Erlernen einer Fremdsprache hilfreich sein können und schnell, möglichst ohne Ausnahme, anwendbar sind. Die Vermittlung tieferen Verständnisses für die Problematik und für die Einordnung von bestimmten sprachlichen Phänomenen ist in vielen dieser Schulgrammatiken nicht beabsichtigt. So spielt es, kann argumentiert werden, für das Erlernen einer Fremdsprache das Wissen darüber eigentlich keine Rolle, ob die Oberfeldumstellung im Deutschen wie in ..., weil er sie hat sehen können durch ein lexikalisches Merkmal bestimmter Verben zustande kommt, oder durch die Veränderung des Werts beim Parameter "Verzweigungsrichtung" in deutschen Verbalkomplexen. Das sind Dinge, die gemeinhin beim Verfassen von didaktischen Grammatiken als nicht relevant betrachtet werden.

Sehen wir uns einmal ein Schema von Wirkungszusammenhängen von Grammatiken an, formuliert von Helbig (1981, 49ff.): 
Grammatik A:

ist der Sprache inhärent, unabhängig von ihrer Erkenntnis

Grammatik $B_{1}$ Linguistische Beschreibung der Grammatik A<smiles>C=CC=CC</smiles>

Grammatik $B_{2}$ :

Didaktische Grammatik:

$$
\mid
$$

Grammatik C:

Lernergrammatik

Abb.1

Welke (1985: 71, zitiert nach Reinecke 1989: 22) fügt noch eine weitere Grammatik hinzu, nämlich die "wissenschaftliche (linguistische oder psychologische) Beschreibung der Grammatik C als Grammatik D".

Für die Sprachdidaktik stellt sich das Problem der "didaktischen Brechung dieser linguistischen Abbildung" (ibd.: 21), mit anderen Worten die Frage, wie Grammatik $\mathrm{B}_{2}$ aus Grammatik B ${ }_{1}$ entsteht. Günther (1998: 26ff) betrachtet als Aufgabe der Sprachdidaktik die Klärung und Vermittlung der sprachlichen Sachverhalte. Die didaktische Aufbereitung Günther spricht von "Transformation" - dürfe nicht von einer "reduzierte[n] MickeymouseVersion" (ibd.: 27) einer Sprache und ihrer Grammatik ausgehen. Sprachliche Phänomene müssen nach Günther in ihrer Komplexität angemessen erfasst werden. Die intensive Beschäftigung mit sprachwissenschaftlichen Theorien sollte somit ein Bestandteil der Ausbildung der künftigen Lehrenden werden. Dies bedeutet eine Erweiterung der Auffassung von didaktischer Grammatik: Günther plädiert, wie ich ihn verstehe, dafür, dass sprachwissenschaftliche Erklärungen, die Betonung liegt auf "wissenschaftlich", didaktisch aufbereitet werden, denn nur, wenn in die Tiefe gehende Analysen miteinbezogen werden, können die entsprechenden Sachverhalte auch angemessen erfasst und überzeugend vermittelt werden. 


\section{$3 \quad$ Grammatik im Unterricht}

\subsection{Einwände und Fragen}

In diesem Zusammenhang stellt sich die Frage, wieviel Grammatik man einem Lerner (einer Fremd- oder der Muttersprache) und ab welchem Lebensalter man sie ihm zumuten kann und sollte? Renkl (1996) vertritt die Auffassung, dass grammatisches Wissen, wenn es beispielsweise isoliert und in keinem Anwendungs- und Sinnkontext vermittelt wird - Renk1 zufolge gibt es noch weitere hemmende Gründe - , "träges Wissen" sei.

Ein weiteres Problem könnte darin bestehen, dass "Grammatikunterricht vor dem 12. Lebensjahr ein unfruchtbarer Versuch am noch untauglichen Subjekt" sei (Gaiser 1950 in Rötzer 1983: 3). Dies liegt in der Gedankenlinie Piagets, dem zufolge so etwa um das 11./12. Lebensjahr der Übergang von der "Phase der konkreten Operationen", des empirischinduktiven Denkens, zur Phase der formalen Operationen", des hypothetisch deduktiven Denkens stattfinde (Piaget 1975). Ingendahl (1999: 140) plädiert dafür, vor allem in der Grundschule z.B. beim Schreibenlernen die "induktiven Regelbildungsversuche" der Kinder zu nutzen und Eisenberg et.al. (1994: 24) fordert den systematischen Rechtschreibunterricht insbesondere erst dann durchzuführen, wenn entwicklungspsychologisch die Zeit dafür reif sei.

Beklagt wird auch häufig, dass grammatisches Wissen, wenn es denn schon beispielsweise in der Grundschule vermittelt wird, nicht korrekt vermittelt werde (cf. auch Haueis 1998: 33f.), etwa bei der Bestimmung der Wortarten. Jeder, der schulpflichtige Kinder hat, weiß, dass in der Tat z.B. Verben als "Tuwörter" bezeichnet werden. Wenn die Kinder feststellen, dass dies längst nicht immer stimme, - so tut der Regen nichts, wenn er fällt - könne die Belegung mit diesen, falschen, Termini sich als hemmend für eine "angemessene theoretische Sprachreflexion" erweisen (Ingendahl 1999: 139f.).

\subsection{Gegeneinwände}

Alleiniger Erkenntnisgewinn durch Induktion kann, wie ich meine, in empirisch erfahrbaren Dingen wie Sprache durchaus problematisch sein. Können sämtliche relevanten Fälle von den Lernenden erschlossen werden und ist bei allen ein qualitativ ausreichender Sprachinput vorhanden? Die Tatsache, dass "induktive Regelbildungsversuche" der Kinder genutzt werden, schließt nicht aus, dass der Sprachunterricht auch deduktive Phasen haben darf.

Die Frage ist, wie die Anhäufung von "trägem Wissen" vermieden und aktives, prozedurales, Wissen aufgebaut werden kann. Im Unterricht finden wir die Situation vor, dass die meisten Sprachlerner, auch in der Grundschule, prozedurales Wissen bereits erworben haben, das sie täglich stundenlang ausüben, indem sie sprechen. Dennoch sollte es ein Ziel auch des Unterrichts in der Muttersprache sein, den Lernprozess zu kanalisieren und zu fokussieren auf Dinge, die sich, warum auch immer, induktiv vielleicht nicht so leicht erschließen lassen.

Noch einmal zurück zu der Behauptung, grammatisches Wissen sei "träge". Dies ist, wie eben bereits erwähnt, u.a. dann so, wenn der Anwendungskontext nicht oder ungenügend vermittelt 
wird bzw. den Lernenden unklar bleibt. So kommt es darauf an, in welchem Zusammenhang und wie grammatisches Wissen gelehrt wird bzw. welche Auffassung von Sprache die Sprachdidaktik hat. Schauen wir nach bei Bühler, dann lesen wir, dass "Sprache wie andere Geräte des Lebens ein menschliches Zweckgebilde ist" (Bühler 1934/1978: 126), sie ist eine zweckgerichtete Handlungsform.

Da Menschen, und also auch Lerner, mit Sprache handeln können, liegt es nahe, im Unterricht darauf, d.h. auf die handlungspraktische Seite von Sprache, Bezug zu nehmen. Denn die Überlegungen "ich tue etwas mit Hilfe von Sprache (z.B. beschreiben, fragen, schimpfen, wünschen usw.), aber wie, d.h. mit welchen sprachlichen Mitteln, tue ich es ?" geben den Anstoß zu einer Reflexion über die Sprache in ihrer Funktion als "handlungspraktisches Bewusstsein" (Redder 1998) .

Dies ist der Ansatzpunkt von funktional konzipiertem Grammatikunterricht (cf. Haueis 1998), der auf die Funktionalität von Sprache fokussiert, ausgehend von der Doppelfrage was kann ich mit Sprache tun und wie kann ich das tun?

\section{Syntaktisch-semantische Einordnung der Modalverben}

Zuerst eine informelle Klärung des Terminus "Modalität": Sie schafft bestimmte Redehintergründe, Diskurse, die als Rahmen für Äußerungen, dienen. Wenn ein Satz eine bestimmte Modalität zugewiesen bekommt, hat der Sprecher eine Intention, die über das ledigliche Feststellen eines Sachverhalts hinausgeht. Die Äußerung wird modifiziert. (Austin 1962) bezeichnete die spezifische kommunikative Rolle einer selbstständigen Äußerungsform als "Illokution" mit Funktionen wie befehlen, behaupten, bitten, danken, fragen oder grüßen. Sätze mit Modalverben sind somit Illokutionen eines bestimmten Typs. In Zifonun et al. (1997) werden i. epistemische, ii. normative, iii. teleologische, iv. volitive und v. circumstantielle Modalverben unterschieden, mit denen der Sprecher i. Einschätzungen, ii. bestimmte Normen, iii. Wünsche, iv. Berücksichtigung der Umstände und v. absolute Notwendigkeiten zum Ausdruck bringt.

\section{$5 \quad$ Auswahl der Theorie und ein erstes Beispiel}

Um mich dem o.g. Ziel, nämlich der adäquaten Erfassung sprachlicher Sachverhalte und ihrer Veranschaulichung, zu nähern, muss zuerst einmal eine Theorie ausgewählt werden. Ich möchte hier auf das Grammatik Pieter Seurens, die 'Semantische Syntax' (Seuren 1996), zurückgreifen. Sie erfüllt zwei Kriterien als wichtige Bedingung für den Typ der Veranschaulichung, die im Folgenden dargestellt wird: Die Semantische Syntax ist transformationell und bedient sich bei der Satzgenerierung bzw. -analyse einer kompakten Menge an Regeln, d.h. sie kommt mit relativ wenigen Regeln aus.

Einige wenige Erläuterungen zur Theorie sollen hier ausreichen: Jeder sprachlichen Äußerung wird in der Semantischen Syntax eine Tiefenstruktur zugeordnet. Diese repräsentiert die Bedeutung der Äußerung und hat, ganz gleich um welche Sprache es sich handelt, stets einen identischen Aufbau. Bewegungsregeln überführen die Tiefenstruktur schließlich in eine 
Oberflächenstruktur. Das ist die syntaktisch wohlgeformte Äußerung mit der linearen Anordnung der Wörter, wie sie uns im Sprachgebrauch begegnet.

Betrachten wir ein Beispiel, nämlich den Satz ich will Rugby spielen. Ich werde bei der sprachwissenschaftlichen Ableitung diejenigen Details, die nicht unbedingt für das prinzipielle Verständnis der folgenden Ideen notwendig sind, weglassen. Die (stark vereinfachte) Tiefenstruktur ${ }^{1}$ könnte so aussehen:

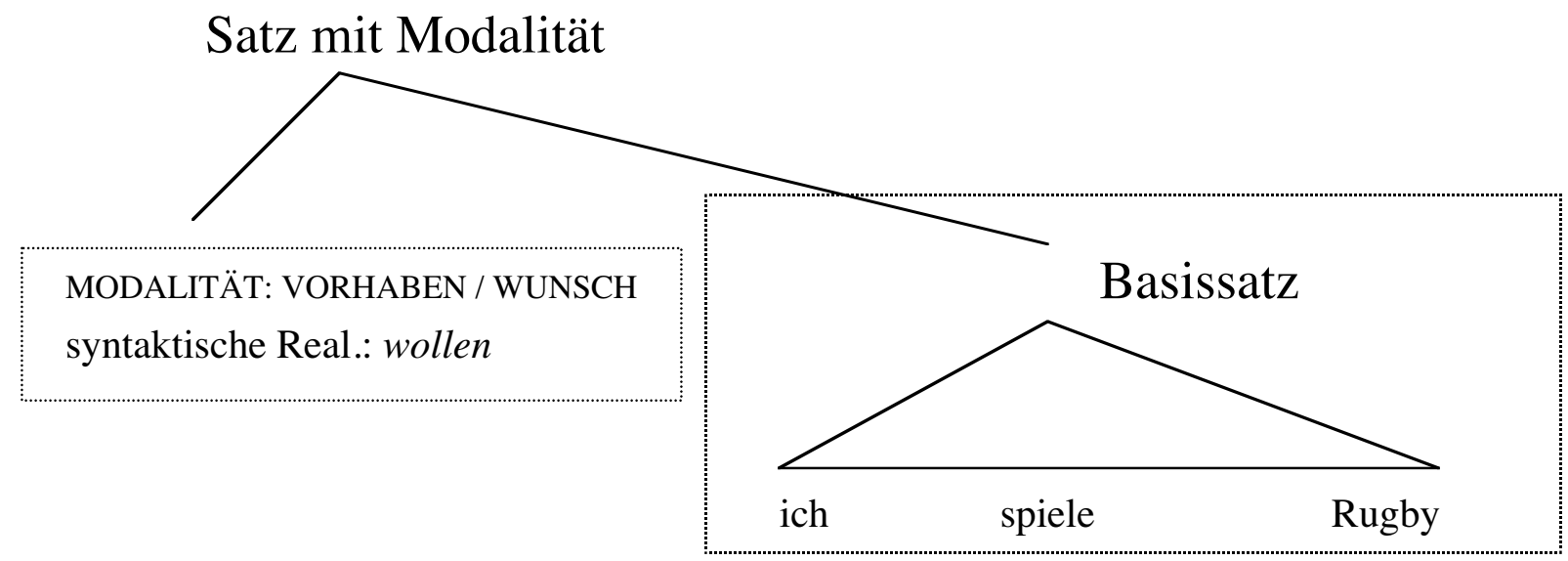

Abb. 2

Im Basissatz wird gesagt, dass jemand (der Sprecher) Rugby spielt. Diese Aussage wird durch das Modalverb dahingehend modifiziert, dass der Sprecher vorhat bzw. den Wunsch äußert, Rugby zu spielen. Die Äußerung im Basissatz und die durch das Modalverb modifizierte Äußerung haben jeweils unterschiedliche Wahrheitsbedingungen: Ich spiele Rugby ist wahr dann, wenn der Sprecher zu einem bestimmten Zeitpunkt und an einem bestimmten Ort Rugby spielt. Ich will Rugby spielen ist jedoch dann wahr, wenn der Sprecher zu einem bestimmten Zeitpunkt und an einem bestimmten Ort vorhat, Rugby zu spielen. Das Modalverb verändert die Wahrheitsbedingungen und damit die Bedeutung des Satzes. Man kann es so sehen, dass Modalität, die sich syntaktisch als Modalverb äußert und Wunsch, Vermutung, Erlaubnis, Befehl oder Einschätzung ausdrücken kann, ein Wirkungselement ist, das, bildlich gesagt, über der Aussage schwebt. Solche Wirkungselemente werden zuweilen auch als Operatoren bezeichnet. Durch eine bestimmte Bewegung wird das Modalverb mit dem Verb im Basissatz verbunden und in unserem Falle entsteht die verbale Gruppe spielen wollen. Die folgenden Abbildungen sollen diese Vorgänge verdeutlichen:

\footnotetext{
1 Die in Abb. 2 gezeigte Tiefenstruktur weist einen grundlegenden Unterschied zu derjenigen in Seurens Grammatik auf. Im Gegensatz zu letzterer hat sie im Basissatz - Seuren spricht von lexikalem Nukleus - die Abfolge Subjekt - Verb - Objekt (SVO). In der Semantischen Syntax haben Tiefenstrukturen dagegen stets die Abfolge Prädikat - Subjekt - Verb, in diesem Fall also Verb - Subjekt - Objekt (VSO).
} 


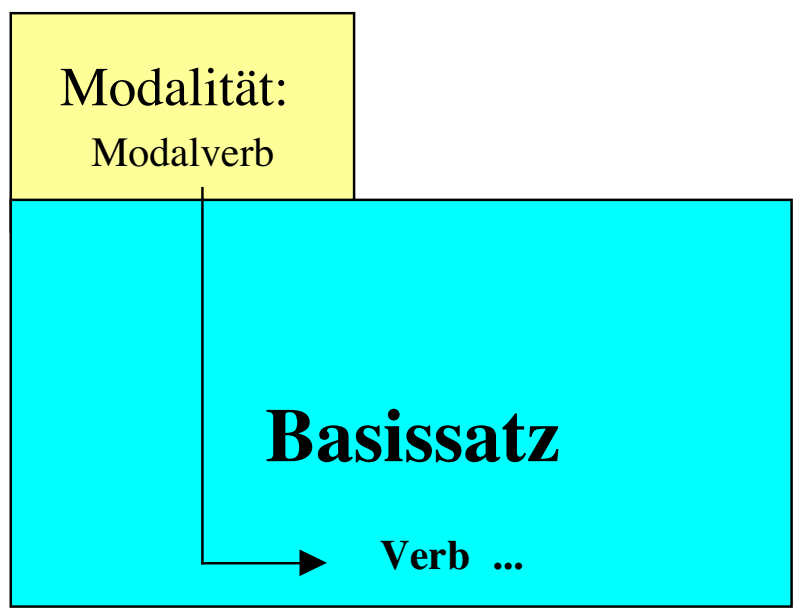

Abb.3

Das Modalverb, der modale Operator, wird in die Basisäußerung herabgesenkt und mit dem Verb verbunden, woraus sich in dem Fall die Gruppe spielen wollen ergibt.

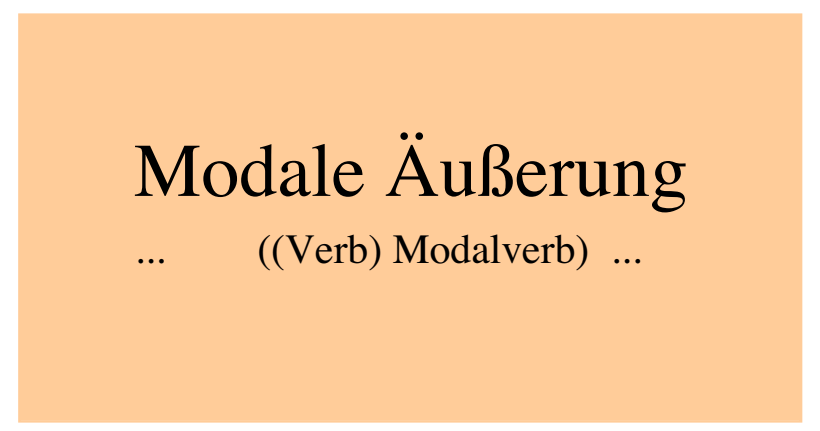

Abb. 4

Übertragen auf den Beispielsatz können die oben beschriebenen Mechanismen konkretisiert werden: 

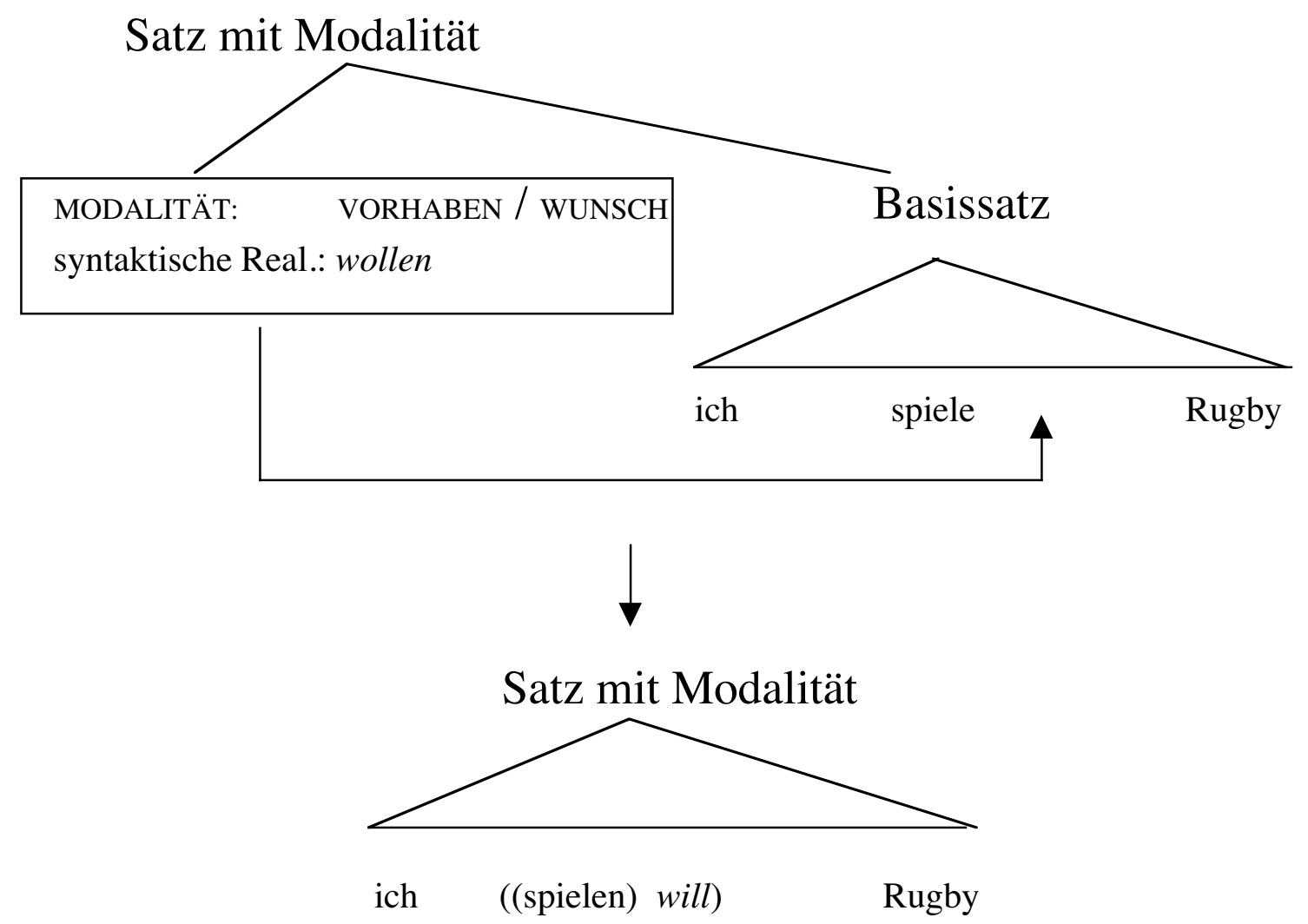

Abb. 5

Zuletzt wird noch der nicht-finite Teil der verbalen Gruppe an das Satzende versetzt:

\section{Satz mit Modalität}

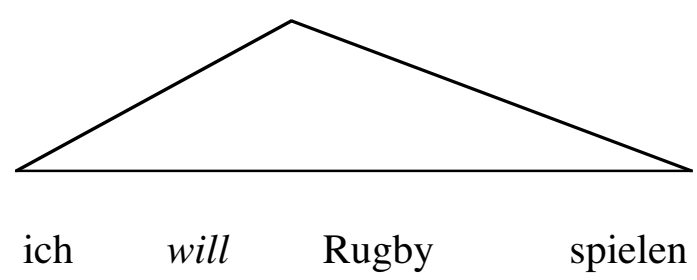

Abb. 6

Was ich gerade versucht habe zu zeigen, wird in der Terminologie bestimmter theoretischer Schulen als "Operator-Lowering" bezeichnet. Es geht jetzt nicht darum, eventuell mit Ausnahme von Schülern der Sekundarstufe II oder Studierenden einer einschlägigen Fachrichtung, die Lernenden mit diesem Terminus, geschweige denn mit den "technischen" Details der Ableitung eines Satzes zu belasten. Jedoch bietet, wie ich finde, die Vorstellung eines Operators, der in der Tiefenstruktur als über dem eigentlichen Satz schwebendes Wirkelement dargestellt wird, einen fruchtbaren Ansatz zu einer Veranschaulichung und Begrifflichmachung des Konzepts "Modalität". 


\section{Veranschaulichung}

Im Rahmen der Behandlung von Modalität im Unterricht halte ich das Niveau der Veranschaulichung geeignet für Schüler der 5./6. Klasse. Um den Einstieg in das doch ziemlich abstrakte Thema "Modalität und den Aufbau des Konzept "Modalität" zu erleichtern, könnte es sinnvoll sein, die Veranschaulichung, die darin besteht, dass die Schüler Modalität auch visuell erfahren, bereits zu Anfang der Unterrichtssequenz anzubieten. Vielleicht ist es im Sinne des Lernens durch Induktion geboten, dass die Schüler zuerst beschreiben, was sie sehen und dann ihre Überlegungen auf semantische Aspekte ausweiten. Das erworbene Konzept von Modalität kann dann im weiteren Verlauf der Unterrichtssequenz vertieft und gefestigt z.B. durch die Produktion von Texten.

Als Beispiele sollen im Folgenden Verkehrsschilder dienen. Sie stellen allgegenwärtige Manifestationen von Modalität in unserem Alttag dar und sind im Erfahrungsbereich der Schüler verankert.

In Abb. 7 werden mit Hilfe von Schildern Aussagen gemacht, die von einem modalen Operator modifiziert werden. Betrachten wir folgendes (Phantasie)-Schild:

Abb. 7

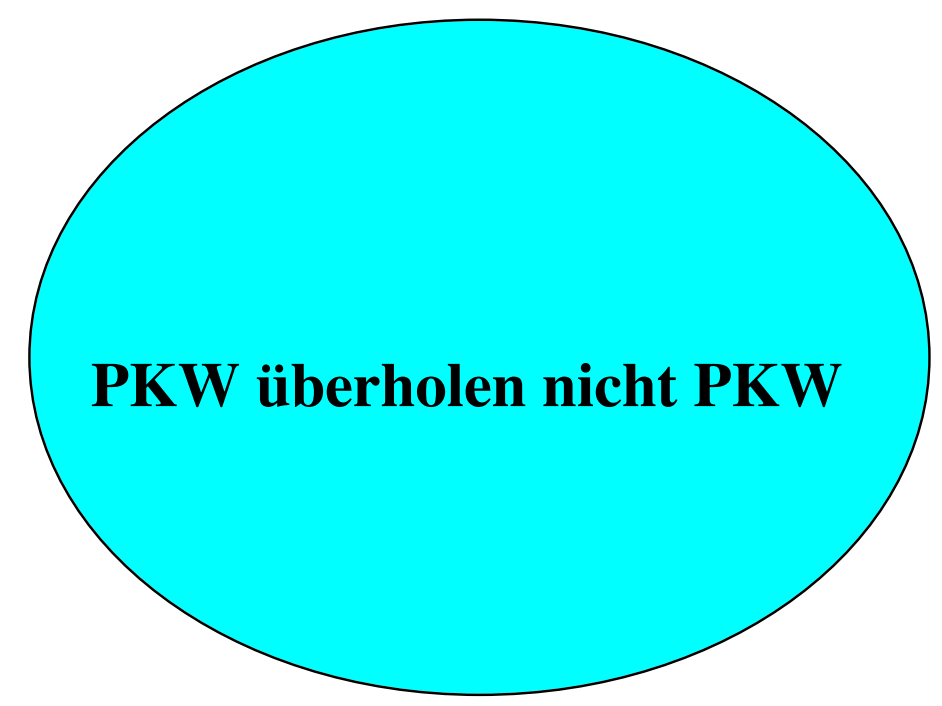

Die Aussage lautet: "PKW überholen nicht PKW". Jetzt stelle man sich folgendes Schilderensemble vor: 


\section{dürfen}

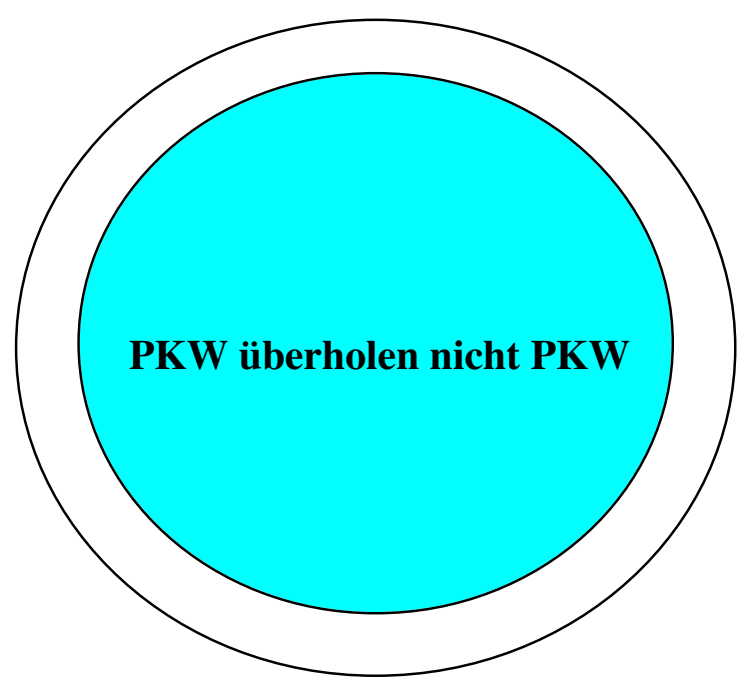

Abb. 8

Der Modaloperator hat Einfluss auf die Aussage und befindet sich in dem gelblichen Kästchen über der Basisaussage, ganz ähnlich wie in der oben skizzierten Tiefenstruktur.

Wenn der Modaloperator in die Äußerung wandert (9)a - dies ist dann "Operator-Lowering" ergibt sich das folgende Verkehrsschild, das im Straßenverkehr anzutreffen ist und ein Überholverbot für PKW ausspricht (9)b:

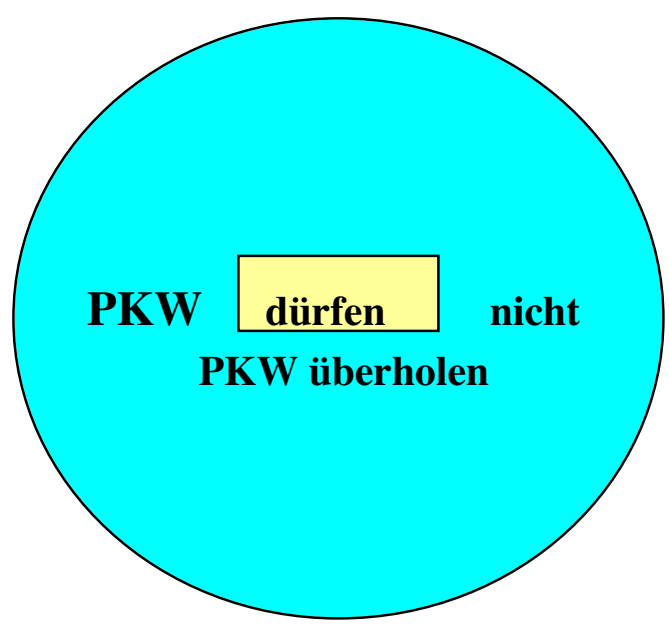

Abb. (9)a 


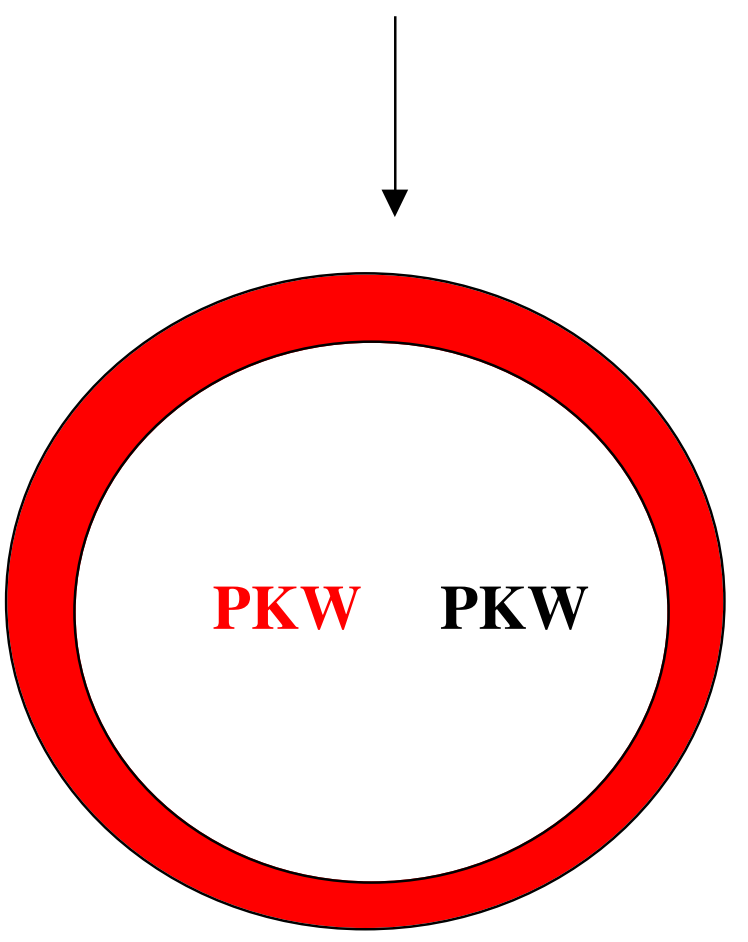

Abb. (9)b

Es wäre anschließend sinnvoll, die Schüler die Art der Modalität ("nicht dürfen"), die durch dieses Zeichen ausgedrückt wird, genauer bestimmen zu lassen ("Verbot") und danach selbst Veranschaulichungen zu erarbeiten, um zu überprüfen, ob das Prinzip der Modifizierung von Aussagen durch einen modalen Operator erfasst wurde.

Ein weiteres Beispiel: In Abb. 10 lautet die Aussage in dem in dem weißen Schild: "Ich trage einen Helm". Der modale Operator müssen befindet sich über der Basisaussage:
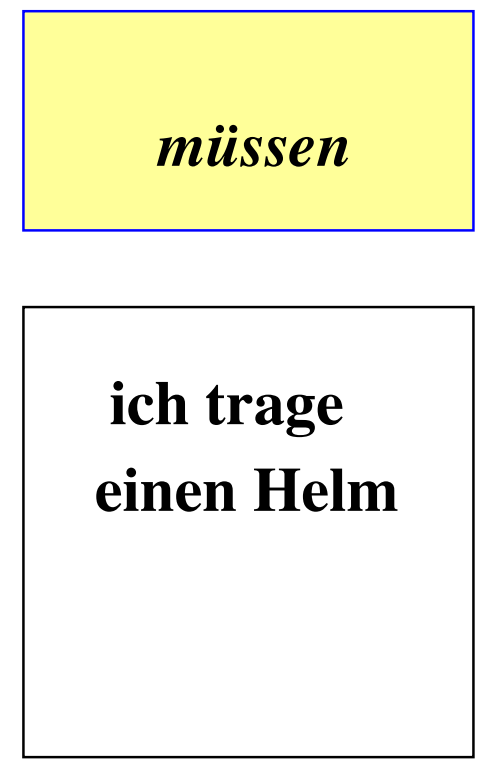

Abb. 10 
Operator-Lowering führt hier zu einem Gebotsschild, das man z.B. auf Baustellen findet und Helmpflicht anzeigt.:

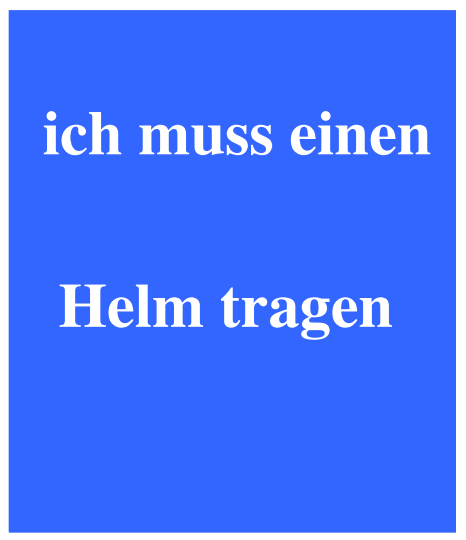

Abb. 11

Zur besseren Veranschaulichung könnte die Aussage anstatt in natürlicher Sprache auch als ikonisches Zeichen (Darstellung eines Helms) wiedergegeben werden.

Für den Unterricht ließen sich Beispiele finden, die andere Modalitätsbereiche abdecken. So hat das Auxiliar des Futurs werden modale Potenz. Stellen wir uns Tagespläne vor, bei denen eine Uhr als Symbol für einen modalen Operator über einer Äußerung verwendet werden kann.
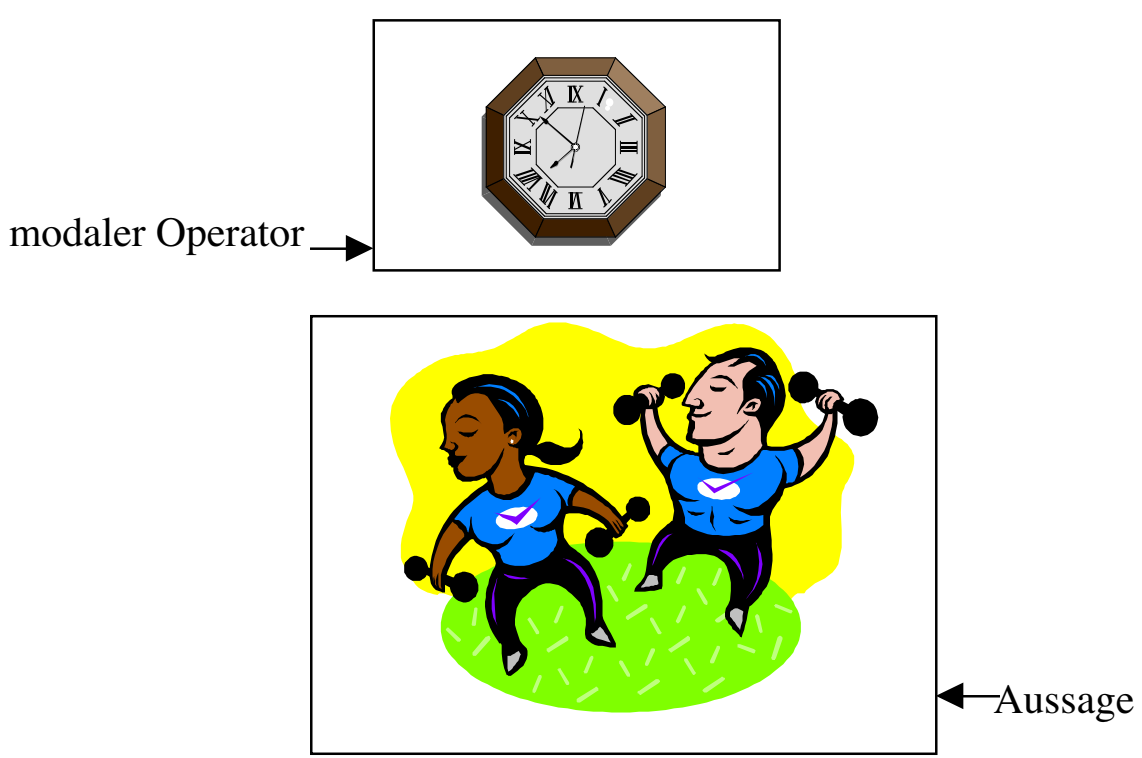

Abb. 12 
Die Basisaussage in diesem Ensemble laute "die Schüler treiben Sport". Durch OperatorLowering kommt die Modalität (und in diesem Fall auch die Zeitangabe) in die Äußerung, so dass die Aussage in Abb. 13 so wiedergegeben werden kann: "die Schüler werden (um 10 vor 8) Sport treiben".

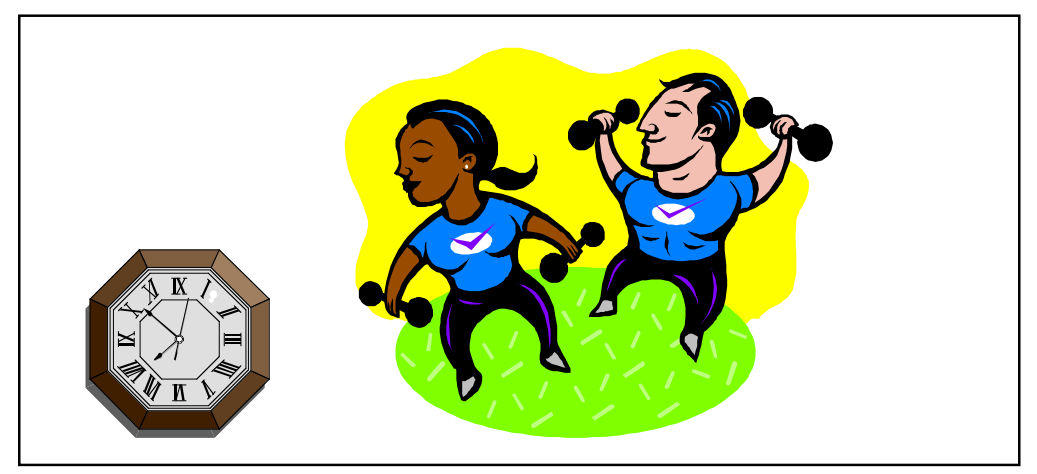

Abb. 13

Wenn wir noch einmal zu unserer Theorie zurückgehen, so könnte eine Tiefenstruktur für obiges Beispiel folgendermaßen aussehen:

\section{Satz mit Modalität}

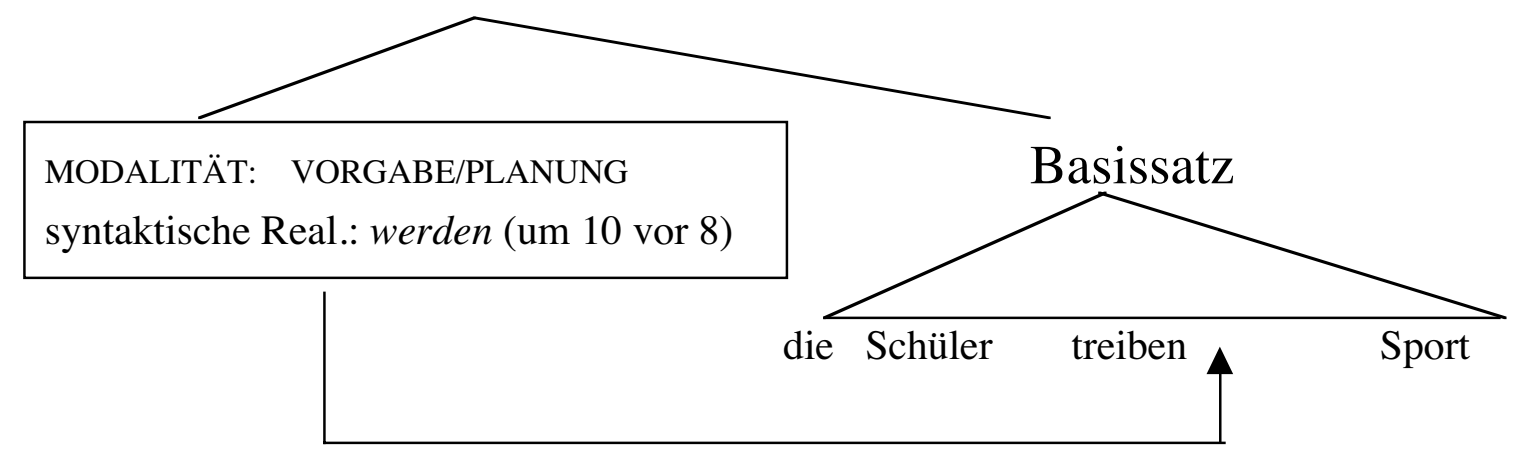

Abb. 14

Deutlich ist die strukturelle Gleichheit zwischen der Veranschaulichung mit Hilfe von Symbolen in Abb. 12 und der Repräsentation durch die Semantische Syntax. Die Theorie, die bei der Erfassung von Details rasch an Komplexität gewinnt, geht von einfachen Grundgedanken aus, die sich gut didaktisch reduzieren und veranschaulichen lassen. Ich möchte noch darauf hinweisen, dass nicht die hier verwendete Theorie an sich die entscheidende Rolle spielt - schließlich sollen die Schüler nicht zu Sprachtheoretikern ausgebildet werden - sondern es geht um das Erfassen und Begreifbarmachen von sprachlichen Strukturen. 


\section{$7 \quad$ Fazit}

Ich habe in diesem Aufsatz anhand der Modalverben und des Konzepts der Modalität zu zeigen versucht, dass grammatisches Wissen, das explizit im Unterricht vermittelt wird, nicht zwangsläufig träges Wissen ist. Es wird dann prozedural, wenn die Lernenden beginnen, den funktionalen Charakter von Sprache, auch mit Hilfe von Reflexion, zu erkennen, dadurch dass sie mit Sprache handeln bzw. durch Sprache zum Handeln bewegt zu werden. Letzteres geschieht zum Beispiel, wenn Aufforderungen, Gebote und Verbote, die auf Schildern ausgedrückt sind, interpretiert werden. Aber auch Wünsche oder Vermutungen, etwa durch Gebrauch von werden, können symbolisch repräsentiert werden und funktionalen Charakter von Sprache verdeutlichen.

Eine sprachwissenschaftliche Theorie lieferte in diesem Aufsatz die Grundideen für die Veranschaulichung und die didaktische Reduzierung. Es ist im Allgemeinen der Fall, dass die Basisannahmen guter und solider Theorien einfach und plausibel sind. Sie haben das Ziel, das sprachliche System transparent zu machen. Die Aufgabe für die Autoren von Lehrwerken und auch für Lehrkräfte muss dann im Wesentlichen darin bestehen, sprachwissenschaftliche Erklärungen (ausgewählter Theorien) didaktisch zu transformieren und zu reduzieren.

\section{Literaturangaben}

Austin, John Langshaw (1962): How to do things with words. Oxford.

Bühler, Karl (1934): Sprachtheorie. Jena.

Bühler, Karl (1978): Sprachtheorie. 3.Aufl. Stuttgart.

Eisenberg, Peter/Spitta, Gudrun/Voigt, Gerhard (1994): "Schreiben: Rechtschreibung". Praxis Deutsch 124: 14-25.

Gaiser, Konrad (1950): "Wieviel Grammatik braucht der Mensch". In: Rötzer, Hans Gerd. (ed.) (1973): Zur Didaktik der deutschen Grammatik. Darmstadt: 1-15.

Günther, Hartmut. (1998): "Sprachwissenschaft und Sprachdidaktik - Am Beispiel kleiner und großer Buchstaben im Deutschen". Didaktik Deutsch: 17-32.

Haueis, Eduard (1998): "Wie einfach ist der einfache Satz?". Didaktik Deutsch 4: 33- 42.

Helbig, Gerhard (1981): Sprachwissenschaft - Konfrontation - Fremdsprachenunterricht. Leipzig.

Ingendahl, Werner (1999): Sprachreflexion statt Grammatik. Ein didaktisches Konzept für alle Schulstufen. Tübingen (= Reihe Germanistische Linguistik 211).

Piaget, Jean (1975): Der Aufbau der Wirklichkeit beim Kinde. ges. Werke Bd. 2. Stuttgart.

Redder, Angelika (1998): "Sprachwissen als handlungspraktisches Bewusstsein - eine funktional-pragmatische Diskussion". Didaktik Deutsch: 60-76.

Reinecke, Werner (1989): "Theorie des Fremdsprachenunterrichts: Disziplinen - Gegenstände - Konzepte". In: Buscha, Joachim/ Schröder, Jochen: Linguistische und didaktische Grammatik. Beiträge zu Deutsch als Fremdsprache. Leipzig (= Linguistische Studien, Festschrift zum 60. Geburtstag von Gerhard Helbig): 11- 29.

Renkl, Alexander (1996): "Wenn Erlerntes nicht genutzt wird". Psychologische Rundschau 47: 78-92. 
Seuren, Pieter (1996): Semantic Syntax. Oxford.

Welke, Klaus (1985): "Fremdsprachenunterricht und psychologische Realität der Grammatik". In: Nyholm, Kurt: Grammatik im Unterricht. Dritte Sprachwissenschaftliche Konferenz Finnland-DDR, September 1984. Akademi Forskninginst. Abo.

Zifonun, Gisela et al. (1997): Grammatik der deutschen Sprache. 3 Bde. Berlin/New York. 